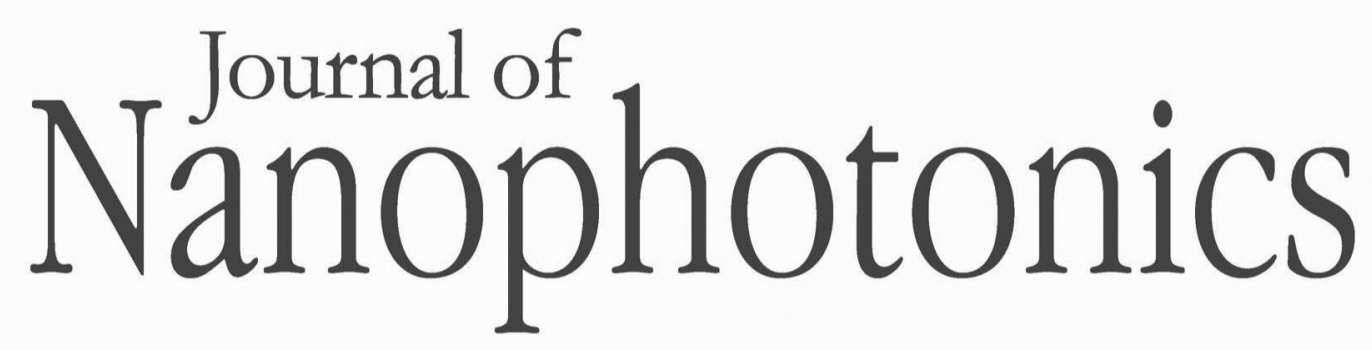

\title{
Mouse neuroblastoma cell-based model and the effect of epileptic events on calcium oscillations and neural spikes
}

\author{
Suhwan Kim \\ Unsang Jung \\ Juyoung Baek \\ Sangwon Lee \\ Woonggyu Jung \\ Jeehyun Kim \\ Shinwon Kang
}

\section{O SPIE}




\title{
Mouse neuroblastoma cell-based model and the effect of epileptic events on calcium oscillations and neural spikes
}

\author{
Suhwan Kim, ${ }^{\text {a }}$ Unsang Jung, ${ }^{a}$ Juyoung Baek, ${ }^{\text {a }}$ Sangwon Lee, ${ }^{\text {a }}$ \\ Woonggyu Jung, ${ }^{b}$ Jeehyun Kim, ${ }^{\mathrm{c}}$ and Shinwon Kang ${ }^{\mathrm{c}}$ \\ ${ }^{a}$ Kyungpook National University, School of Electrical Engineering and Computer Science, \\ Daegu 702-701, Republic of Korea \\ ${ }^{b}$ Ulsan National Institute of Science and Technology, School of Nano-Bioscience and \\ Chemical Engineering, UNIST-gil 50(100 Banyeon-ri), Eonyang-eup, Ulju-gun, \\ Ulsan Metropolitan City 689-798, Republic of Korea \\ ${ }^{\mathrm{c}}$ Kyungpook National University, College of IT Engineering, School of Electronics Engineering, \\ 1370 Sankyuk-dong, Buk-gu, Daegu 702-701, Republic of Korea \\ jeehk@knu.ac.kr or swkang@knu.ac.kr
}

\begin{abstract}
Recently, mouse neuroblastoma cells have been considered as an attractive model for the study of human neurological and prion diseases, and they have been intensively used as a model system in different areas. For example, the differentiation of neuro2a (N2A) cells, receptor-mediated ion current, and glutamate-induced physiological responses have been actively investigated with these cells. These mouse neuroblastoma N2A cells are of interest because they grow faster than other cells of neural origin and have a number of other advantages. The calcium oscillations and neural spikes of mouse neuroblastoma N2A cells in epileptic conditions are evaluated. Based on our observations of neural spikes in these cells with our proposed imaging modality, we reported that they can be an important model in epileptic activity studies. We concluded that mouse neuroblastoma N2A cells produce epileptic spikes in vitro in the same way as those produced by neurons or astrocytes. This evidence suggests that increased levels of neurotransmitter release due to the enhancement of free calcium from 4-aminopyridine causes the mouse neuroblastoma N2A cells to produce epileptic spikes and calcium oscillations. (C) 2013 Society of Photo-Optical Instrumentation Engineers (SPIE) [DOI: 10.1117/1.JNP.7.073794]
\end{abstract}

Keywords: mouse neuroblastoma cell; epilepsy; calcium oscillation; neural spikes.

Paper 13056SSP received Jul. 18, 2013; revised manuscript received Sep. 26, 2013; accepted for publication Oct. 3, 2013; published online Nov. 5, 2013.

\section{Introduction}

Since about the turn of the 21 st century, several chronic diseases associated with the brain, such as Parkinson's disease, dystonia, chronic pain, and epilepsy, have become an issue. In North America, the reported epilepsy incidence is approximately 50/100,000 people per year. ${ }^{1}$ Epilepsy is a condition that is characterized by abnormal electrical activities in the brain. These seizures are caused by an electrical storm of abnormal neuronal activities that spread from the locus (or point of origin) to adjacent tissue.

In order to understand the mechanism underlying the generation of epilepsy, it is important to build an effective model that can produce epileptic spikes in vitro or in vivo. Therefore, any newly developed drug should be applied to those models in order to confirm their feasibility. Previously, researchers have made significant contributions in developing several epileptic models. Luytton has explained in detail the computer-based modeling of epileptic activity. ${ }^{2}$ Sunderam et al. and Albert have described other epilepsy models in their research articles. ${ }^{3,4}$ However, all of these are computational models that focus on detecting epileptic events.

0091-3286/2013/\$25.00 ㄷ 2013 SPIE 
They cannot be used to understand the mechanism of epilepsy generation or the effects of the drug on them.

Some researchers have tried to generate epilepsy in live animals such as rats or mice. Yang and Rothman have built an epilepsy model for experimentation with 4-aminopyridine (4-AP), which generates epilepsy in rats, and Ziburkus et al. and Libri et al. have used hippocampal and olfactory cortical brain slices to generate and understand the effects of epilepsy. ${ }^{5-7}$ These researchers used Krebs fluid with the following composition (in $\mathrm{mM} / \mathrm{L}$ ): $\mathrm{NaCl}$ (118), $\mathrm{KCl}$ (3), $\mathrm{CaCl}_{2}$ (1.5), $\mathrm{NaHCO}_{3}$ (25), $\mathrm{MgCl}_{2} \cdot 6 \mathrm{H}_{2} \mathrm{O}$ (1), and D-glucose (11) (bubbled with $95 \% \mathrm{O}_{2}: 5 \% \mathrm{CO}_{2}, \mathrm{pH}$ 7.4). They made the solution without magnesium in order to produce the epilepsy. Another method to produce epilepsy is to add $200 \mu \mathrm{M}$ of 4-AP to the Krebs solution. The setback of all three of these methods is the requirement of additional surgical preparation in order to inject the drug into the brain or to extract brain slices.

In order to avoid the surgical process in epileptic models, it is necessary to produce epilepsy in cultured neurons. Cao et al. have described a method to produce epilepsy in cultural cortical neurons. ${ }^{8}$ They have reported that cultures have to be exposed to the magnesium-free medium for $3 \mathrm{~h}$ in order to produce epileptic events in neurons. The drawback of this model is that cortical neurons take more time to fully grow than other neurons such as neuroblastomas or hippocampal neurons. Still, there have been no epileptic models that examine the generation of epilepsy in neuroblastoma cells.

Several neuroblastoma cell lines that originate from a single cell that was isolated from a spontaneously occurring or induced tumor of the nervous system are now available and can be maintained in culture infinitely. ${ }^{9}$ The cultural growth of these cells is faster than that of other neuronal cells. These neuroblastoma cells are also used to study other neural disorders such as transmissible spongiform encephalopathies, which are also called prion diseases. ${ }^{10-12}$ These are caused by prion protein propagation in cells. Cellular prion protein is a plasma membrane protein that is involved in copper uptake, protection against oxidative stress, cell adhesion, differentiation signaling, and survival in the central nervous system. A recent study has shown that the deletion of the prion gene in mice enhances their sensitivity to seizures in vivo and neuronal excitability in vitro. ${ }^{13}$ Ratte et al. have reported that the threshold for epileptiform activity is increased in prion-knockout mice. ${ }^{14}$ Still, much is unknown with respect to prion proteins and epilepsy. Mouse neuroblastoma neuro2a (N2A) cells have been considered one of the best models for studying prion protein effects. Many researchers have also utilized them to study the properties and mechanisms of prion proteins. In order to understand the involvement of prion proteins in epileptic events, it is very important to find a way to induce epilepsy in mouse neuroblastoma N2A cells.

This article describes a mouse neuroblastoma cell-based epileptic model and its use in the analysis of calcium oscillations and neural spikes in seizure events. This is a basic model that can be used to understand epileptic processes in mouse neuroblastoma N2A cells. It also illustrates the calcium oscillations that are produced by these mouse neuroblastoma N2A cells during epileptic events. The results showed that mouse neuroblastoma N2A cells produce epileptic spikes in vitro in the same way as those that are produced by neurons or astrocytes. The results clearly indicate that the release of neurotransmitters was increased with the use of 4-AP, which caused mouse neuroblastoma N2A cells to produce neural spikes and calcium oscillations. The characteristics of the epileptic spikes of mouse neuroblastoma N2A cells were similar to those produced by neurons or astrocytes. We hope that the outcome of this study will provide potential steps toward understanding the role of mouse neuroblastoma N2A cells and 4-AP in the physiological pathway of in vitro epilepsy models.

\section{Materials and Methods}

\subsection{Mouse Neuroblastoma N2A Cell Culture}

The mouse neuroblastoma N2A cells were purchased from American Type Culture Collection (CCl-131, Manassas, Virginia) and cultured at a density of $1.0 \times 10^{4}$ in Dulbecco's modified Eagle's medium (DMEM) that was supplemented with heat-inactivated $10 \%$ fetal bovine 
serum (GIBCO, Life Technologies Corporation, Grand Island, New York) in a $\mathrm{CO}_{2}$ incubator that was supplied with $5 \% \mathrm{CO}_{2}$ at $37^{\circ} \mathrm{C}$. Confluent $(75 \%)$ cells were washed twice with phosphatebuffered saline $(1 \times)$ and digested with $0.25 \%$ trypsin with $1 \times$ TrypleTM Express, which was followed by centrifugation (1500 rpm for $5 \mathrm{~min}$ ) in order to harvest the cells. Subsequently, single-cell suspensions were used to calculate cell number with the standard tryphan blue and the hemocytometer method. After the cell count, we resuspended the cells with media containing $10 \%$ fetal bovine serum and cultured them for 7 days. The DMEM media was changed every 2 days.

\subsection{Extracellular Signal Recording}

In order to prepare the cells for extracellular recording, the cell media (DMEM) was removed from the culture dish, and $5 \mathrm{~mL}$ of Krebs solution [containing (in $\mathrm{mM} / \mathrm{L}$ ): $\mathrm{NaCl}$ (118), $\mathrm{KCl}$ (3), $\mathrm{NaHCO}_{3}$ (25), $\mathrm{MgCl}_{2} \cdot 6 \mathrm{H}_{2} \mathrm{O}, \mathrm{CaCl}_{2}$ (2.5), and d-glucose (11), $\left.\mathrm{pH} 7.4\right]$ was added. ${ }^{15}$ The extracellular signal recording was performed with three electrodes with diameters of $120 \mu \mathrm{m}$. Two electrodes were used for the signal recording and one was used as the reference. The electrodes were separated from the target cells by $1 \mathrm{~mm}$, and the moveable actuator was used to acquire the signal. A 16-channel microelectrode AC amplifier (A-M Systems, Carlsborg, Washington) was used to record the neural signals. The cut-off frequency was set at $100 \mathrm{~Hz}$ for the high-pass filter and at $10 \mathrm{kHz}$ for the low-pass filter, so that it would be able to remove the local field potential from the neural spike signals. The gain of the amplifier was adjusted to 2000.

\subsection{Calcium Imaging with a Confocal Microscope}

A confocal microscope (LSM-700, Carl Zeiss AG, Oberkochen, Germany) was used to record images of epileptic events in the mouse neuroblastoma N2A cells. For the intracellular examination of the neuroblastoma cells, fluo-4 dye was used to detect the calcium signal transport between the cells. Fluo-4 NW Calcium Assay Kits (Life Technologies Corporation) offer a proprietary assay formulation that requires neither a wash step nor a quencher dye. The fluo-4 NW assay results in larger increase in fluorescence intensity than the standard fluo-3 and fluo-4 assays with a wash step and a Molecular Devices' Calcium 3 assay, which uses a quencher dye. Eliminating the wash step results in lower variability and higher $Z$ values than the standard fluo-4 assay, while providing an easier and faster assay. We removed the Krebs solution from the cells, added the fluo- $4 \mathrm{NW}$, as described in the protocol, and incubated the cell culture for $30 \mathrm{~min}$ before recording the images. The fluorescent signals from the specified structures were averaged in order to obtain $F(t) .{ }^{16}$ Baseline fluorescence $\left(F_{0}\right)$ was the average of the entire image under control conditions, and $\Delta F / F$ was calculated as $(\Delta F / F)(t)=\left[F(t)-F_{0}\right] / F_{0}$, which is called the delta function.

\subsection{Induction of Epileptic Events}

We took the 7-day-old cells out of the incubator and removed the media. We then added $5 \mathrm{~mL}$ of the Krebs solution to the cells and put them back in the incubator for $2 \mathrm{~h}$. Then, we added 4-AP to make a 10-mM concentration in the Krebs solution and again incubated them for 30 to $50 \mathrm{~min}$. In this study, two different concentrations of 4-AP were used in order to produce the epilepsy, and the acquired images were then compared with the control condition.

\section{Results}

\subsection{Neural Spike Recording}

The neural spike recording was performed by manually placing three electrodes in the Krebs solution. Figure 1 shows neural spikes from all the mouse neuroblastoma N2A cells. Neural spikes were recorded in the control condition and $30 \mathrm{~min}$ after the injections of 4-AP with concentrations of 10 or $50 \mathrm{mM}$. Figure 1(a) shows the control signal before epilepsy was produced in the cells. The neural spikes had low magnitudes and frequencies. Figure 1(b) shows the neural 


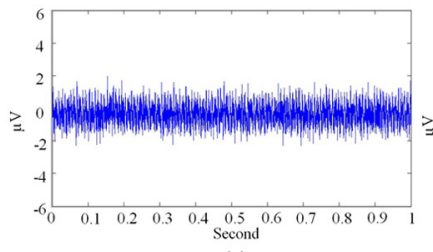

(a)

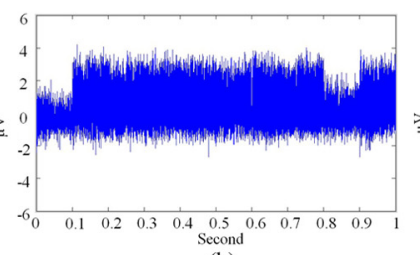

(b)

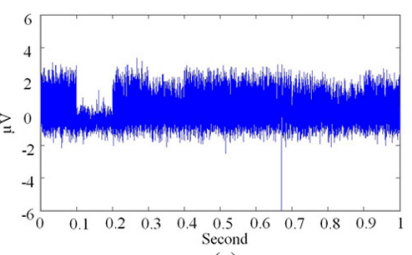

(c)

Fig. 1 Extracellular neural spikes recording: (a) control cells; (b) 30 min after the injection of 4-aminopyridine (4-AP; $10 \mathrm{mM}$ ); and (c) 30 min after the injection of 4-AP (50 mM).

spikes that were acquired $30 \mathrm{~min}$ after the 4-AP $(10 \mathrm{mM})$ injection. The frequency of occurrence and the amplitude of the spikes increased, indicating the onset of epileptic seizure events. The amplitudes of the signal were increased twice compared with the control signal from 2 to $4 \mu \mathrm{V}$. This increment was caused by the 4-AP inhibition of voltage-gated $\mathrm{K}^{+}$channels and an increase in the release of neurotransmitters, such as glutamate, ${ }^{17}$ by 4 -AP. Figure $1(\mathrm{c})$ shows the spike activity $30 \mathrm{~min}$ after the injection of 4-AP $(50 \mathrm{mM})$. The frequency and amplitude of the spike rate were again increased from the control signal but were comparable with that occurring after the 10-mM 4-AP injection.

\subsection{Calcium Imaging}

The imaging of the calcium dynamics was performed with the fluo-4 NW fluorescent dye. Figure 2 shows the calcium dynamics of all of the N2A cells. Figure 2(a) shows the control cells before the production of epilepsy. Less calcium-ion movement was observed in the control cells. Figure 2(b) shows the calcium dynamic images $30 \mathrm{~min}$ after the injection of 4-AP $(10 \mathrm{mM})$. After the injection of the 4-AP in the mouse neuroblastoma N2A cells, a large amount of calcium movement began, indicating the onset of epilepsy. The 4-AP increases the release of neurotransmitters, such as glutamate, and second messengers, such as inositol triphosphate. The large concentration of glutamate can produce epilepsy. ${ }^{18}$ By increasing the concentration of 4-AP to $50 \mathrm{mM}$, large increases in calcium-ion movements were observed [Fig. 2(c)]. The brightness of the spots corresponds to the concentrations of the calcium ions in the specific areas. These results indicated that intensive neural activity occurred, which caused an increased release of neurotransmitters in the extracellular fluid. The transition of a fluorescent spot indicated free calcium-ion movement, which is also known as a calcium oscillation.

\subsection{Delta Function}

The calcium oscillations can be observed by plotting the difference in the electrically excited fluorescent signals from the dormant fluorescent signals, as defined in the calcium imaging with confocal microscopy. Figure 3 shows the delta function of the cells $30 \mathrm{~min}$ after the injections of the 10 or $50 \mathrm{mM}$ concentrations of 4-AP. The shapes of the delta function followed similar
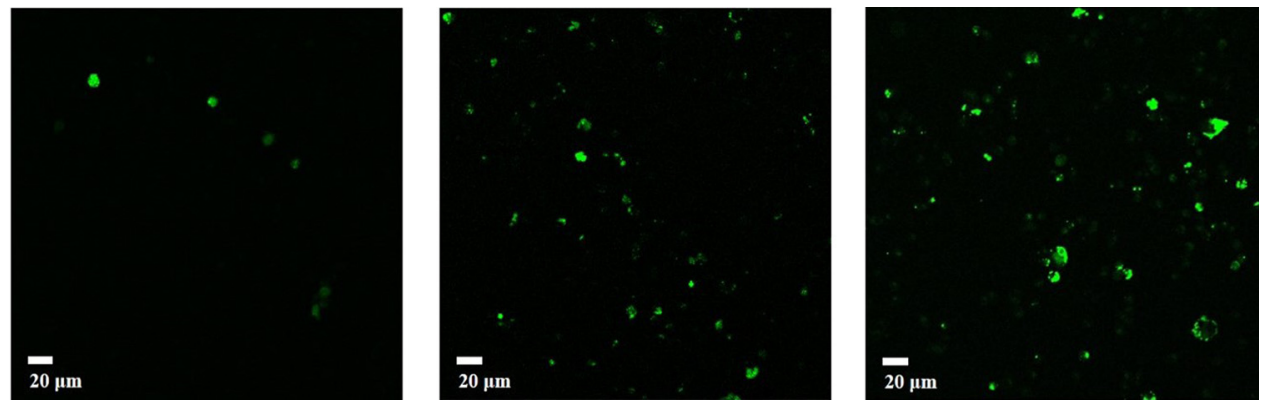

Fig. 2 Fluorescent imaging of mouse neuroblastoma neuro2a (N2A) cells using fluo-4 NW: (a) control cells; (b) 30 min after the injection of 4-AP (10 mM); and (c) 30 min after the injection of 4-AP (50 mM). 


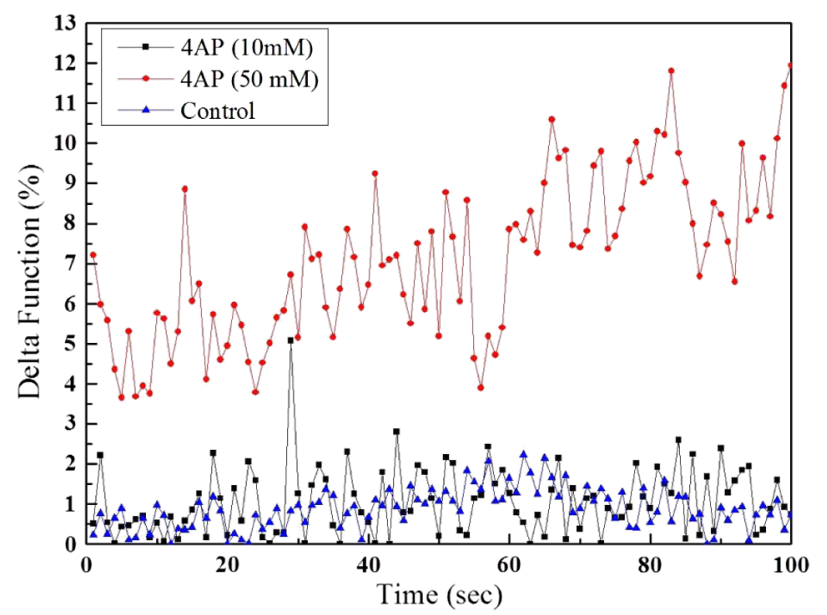

Fig. 3 Calcium oscillations in mouse neuroblastoma N2A cells: control cells (blue line), 30 min after the injection of the 4-AP with $10 \mathrm{mM}$ (black line) and $50 \mathrm{mM}$ (red line).

patterns during epileptic seizure events that have been reported previously by other researchers. Both graphs indicated that calcium signal changes after the injections. The red line in the figure shows the higher amplitude of the delta function with the increased concentration of 4-AP and calcium ions, resulting in higher neural activities. Both cases reflect the onset of epileptic conditions in mouse neuroblastoma N2A cells, because the 4-AP is a $\mathrm{K}^{+}$channel blocker that is widely used to generate epilepsy in the hippocampus, cortical neurons, and astrocytes.

\section{Discussion}

Fluo-4 is a fluorescent dye that gives off an intense signal when it binds with free calcium $\left(\mathrm{Ca}^{2+}\right)$. In control cells, a small amount of $\mathrm{Ca}^{2+}$, which was visualized as bright dots, was observed in the cytosol by the confocal microscopy images [Fig. 2(a)]. After the injections of 4-AP, a large quantity of free $\mathrm{Ca}^{2+}$ was observed in the cytosol as well as in the extracellular fluid, because the mitochondria began to excrete free $\mathrm{Ca}^{2+}$, which was transported outside of the cell through calcium-ion channel activation. Wang and Gruenstein have explained that calcium oscillations start with the increased release of glutamate by each presynaptic membrane. ${ }^{19}$ During the calcium wave propagation, the L-type voltage-gated calcium channels open due to the increased concentration of cytoplasmic calcium. These increased levels of calcium are caused by the increased release of glutamate. Finally, the concentration of free calcium is also increased in the extracellular fluid. During epilepsy, synchronized neural activity occurs, and this tends to increase the release of excitatory neurotransmitters, such as glutamate, and free calcium..$^{20,21}$

Our results indicated that mouse neuroblastoma N2A cells exhibited similar epileptic responses when treated with 4-AP. The increased electrical activity that is shown in Fig. 1 confirmed that 4-AP triggered neural activity in the mouse neuroblastoma N2A cells without any external electric stimulation. No significant electrical signal variations were observed between the two different 4-AP concentrations $(10$ and $50 \mathrm{mM})$ with the extracellular electrical recording. The neural calcium oscillations that were produced by 4-AP were clearly imaged with a confocal microscope when the cells were exposed to fluo-4, as shown in Fig. 2. The bright spots that were considered calcium ions could be traced in the confocal microscopic images, and the occurrence of calcium ions increased in the intracellular and extracellular areas when the cells were under the epileptic state. We found that the propagation of the spots in the confocal microscopic images mostly occurred through axonal and dendritic paths. This observation confirmed that the propagation was indeed due to neural signal transmission. The neural activation that was caused by 4-AP was also evident in the evaluations of the delta function in the somatic region of mouse neuroblastoma N2A cells. For neocortical neurons and astrocytes, Tashiro et al. have already described the use of 4-AP in observations of fast calcium transients during the epileptic condition 
with evaluations of the delta function. ${ }^{22}$ Similar calcium transients from the delta function were observed in the mouse neuroblastoma N2A cells in our study.

\section{Conclusion}

In order to find an alternative cellular model for epilepsy studies, we performed several experiments with mouse neuroblastoma N2A cells. An epileptic condition was induced by the administration of 4-AP into cultured mouse neuroblastoma N2A cells. Without any external electrical stimulation, intensive neural excitation was present in the 4-AP-administered cells, which was confirmed by recording the extracellular electrical potential. We recorded electrical activities in response to two different concentrations of 4-AP $(10$ and $50 \mathrm{mM})$ compared with a control case that was not exposed to 4-AP. Both cases indicated prominent electrical activity in the cells under the epileptic events. A confocal microscope was utilized to visualize the neural activity when the cells were tagged with a fluorescent dye (fluo-4). Later, we plan to apply this model to in vivo animal studies. The confocal microscopic imaging of the cells clearly showed calcium oscillations in the intracellular and extracellular regions. The evaluations of the delta function in the region were consistent with the previously reported results in the literature. Therefore, we concluded that mouse neuroblastoma N2A cells can be a useful model in the study of epilepsy and used as an alternative selection to other neuronal cells or astrocytes. There have been no reports on epilepsy in mouse neuroblastoma N2A cells, and the results in this report suggested a basic model of epilepsy in the cells. However, these results are only from a basic model, and we are now constructing a more detailed model. In the future, by constructing the theoretical bases and comparing them with other neuroblastoma or glioblastoma cells, we will complete a detailed model of epilepsy in neuroblastoma cells.

We expect that our results are important in prion protein studies in which mouse neuroblastoma N2A cells are widely used. As prion proteins can regulate the threshold levels of epileptic events, the use of this model can help to understand the phenomenology and mechanisms of prion proteins in epileptic conditions by analyzing the effects of prion protein in neuroblastoma cells.

\section{Acknowledgments}

This work was supported by the National Research Foundation of Korea (NRF) grant funded by the Korea government (MEST) (No. 2008-0062437) and the commercializing technology project in INNOPOLIS Daegu.

\section{References}

1. http://www.ilae.org/Visitors/Chapters/documents/usa.pdf (2006).

2. W. W. Lytton, “Computer modeling of epilepsy," Nat. Rev. Neurosci. 9(8), 626-637 (2008), http://dx.doi.org/10.1038/nrn2416.

3. S. Sunderam et al., "Stochastic modeling and prediction of experimental seizures in Sprague-Dawley rats," J. Clin. Neurophys. 18(3), 275-282 (2001), http://dx.doi.org/ 10.1097/00004691-200105000-00007.

4. P. S. Albert, "A two-state Markov mixture model for a time series of epileptic seizure counts," Biometrics 47(4), 1371-1381 (1991), http://dx.doi.org/10.2307/2532392.

5. X. F. Yang and S. M. Rothman, "Focal cooling rapidly terminates experimental neocortical seizures," Ann. Neurol. 49(6), 721-726 (2001), http://dx.doi.org/10.1002/(ISSN)1531-8249.

6. J. Ziburkus et al., "Interneuron and pyramidal cell interplay during in vitro seizure like events," J. Neurophys. 95(6), 3948-3954 (2006), http://dx.doi.org/10.1152/jn.01378 .2005 .

7. V. Libri et al., "Effect of filbamate on muscarinic and metabotropic glutamate agonistmedicated responses and magnesium-free or 4-aminopyridine-induced epileptiform activity in guinea pig olfactory cortex neurons in vitro," J. Pharmacol. Exp. Ther. 277(3), 1759-1769 (1996). 
8. H. Y. Cao et al., "Effect of recurrent epileptiform discharges induced by magnesium free treatment on developing cortical neurons in vitro," Dev. Brain Res. 142(1), 1-6 (2003), http://dx.doi.org/10.1016/S0165-3806(03)00005-1.

9. J. B. F. Vandervalk and H. P. M. Vijverberg, "Neuroblastoma cells as possible model in the study of glutamate receptors," Amino Acids 1(1), 91-95 (1991), http://dx.doi.org/10.1007/ BF00808095.

10. J. Solassol, C. Crozet, and S. Lahmann, "Prion propagation in cultured cells," Br. Med. Bull. 66(1), 87-97 (2003), http://dx.doi.org/10.1093/bmb/66.1.87.

11. S. Alais et al., "Mouse neuroblastoma cells release prion infectivity associated with exosomal vesicles," Biol. Cell 100(10), 603-615 (2008), http://dx.doi.org/10.1042/BC20080025.

12. O. Windl et al., "Construction and characterization of murine neuroblastoma cell clones allowing inducible and high expression of the proin protein," J. Gen. Virol. 80(1), 15-21 (1999).

13. R. Walz et al., "Cellular prion protein: implications in seizures and epilepsy," Cell. Mol. Neurobiol. 22(3), 249-257 (2002), http://dx.doi.org/10.1023/A:1020711700048.

14. S. Ratte et al., "Threshold for epileptiform activity is elevated in prion knockout out mice," Neuroscience 179, 56-61 (2011), http://dx.doi.org/10.1016/j.neuroscience.2011.01.053.

15. B. J. Whalley, G. J. Stephens, and A. Constanti, "Investigation of the effects of the novel anticonvulsant compound carisbamate (RWJ-333369) on rat piriform cortical neurons in vitro," Br. J. Pharmacol. 156(6), 994-1008 (2009), http://dx.doi.org/10.1111/(ISSN)1476-5381.

16. N. Kang et al., "Astrocytic glutamate release-induced transient depolarization and epileptiform discharges in hippocampus CA1 pyramidal neurons," J. Neurophysiol. 94(6), 41214130 (2005), http://dx.doi.org/10.1152/jn.00448.2005.

17. K. Alvina and K. Khodakhah, "The therapeutic mode of action of 4-aminopyridine in cerebellar ataxia," J. Neurosci. 30(21), 7258-7268 (2010), http://dx.doi.org/10.1523/ JNEUROSCI.3582-09.2010.

18. K. R. Byrnes, B. J. Loane, and A. I. Faden, "Metabotropic glutamate receptors as targets for multipotential treatment of neurological disorders," Neurotherapeutics 6(1), 94-107 (2009), http://dx.doi.org/10.1016/j.nurt.2008.10.038.

19. X. S. Wang and E. I. Gruenstein, "Mechanism of synchronized $\mathrm{Ca}^{2+}$ oscillations in cortical neurons," Brain Res. 767(2), 239-249 (1997), http://dx.doi.org/10.1016/S0006-8993(97) 00585-4.

20. G.-F. Tian et al., "An astrocytic basis of epilepsy," Nat. Med. 11(9), 973-981 (2005), http:// dx.doi.org/10.1038/nm1277.

21. J. Xu et al., "Glutamate-induced exocytosis of glutamate from astrocytes," J. Biol. Chem. 282(33), 24185-24197 (2007), http://dx.doi.org/10.1074/jbc.M700452200.

22. A. Tashiro, J. Glodberg, and R. Yuste, "Calcium oscillation in neocortical astrosytes under epileptiform condition,” J. Neurobiol. 50(1), 45-55 (2002), http://dx.doi.org/10.1002/neu.v50:1.

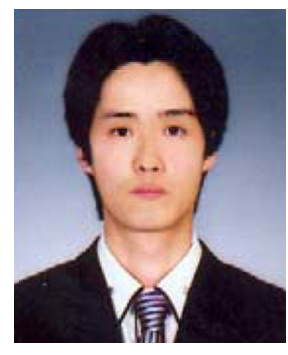

Suhwan Kim received his MS degree in electrical engineering and computer science from Kyungpook National University, Daegu, South Korea. $\mathrm{He}$ is currently working toward the PhD degree in Prof. Shinwon Kang's group at Kyungpook National University. His current research is focused on the study of distributed fiber sensor and multiphoton microscopy.

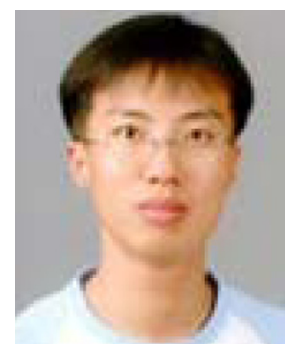

Unsang Jung received his $\mathrm{PhD}$ degree in electrical engineering and computer science from Kyungpook National University, Daegu, South Korea. He is currently working toward the post PhD degree in Prof. Jeehyun Kim's group at Kyungpook National University. His current research is focused on the study of optical coherent tomography and multiphoton microscopy. 


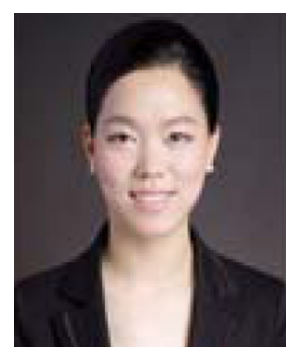

Juyoung Baek received her bachelor's degree in electrical engineering and computer science from Kyungpook National University, Daegu, South Korea. She is currently working toward the MS degree in Prof. Jeehyun Kim's group at Kyungpook National University. Her current research is focused on the study of neuroscience.

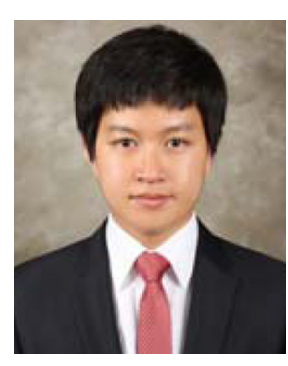

Sangwon Lee received his MS degree in electrical engineering and computer science from Kyungpook National University, Daegu, South Korea. $\mathrm{He}$ is currently working toward the PhD degree in Prof. Shinwon Kang's group at Kyungpook National University. His current research is focused on the study of quantum dots based on LED and organic electronic devices.

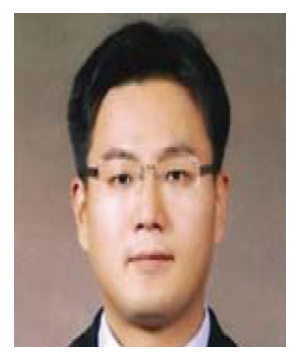

Woonggyu Jung received his $\mathrm{PhD}$ in 2008 from the Department of Biomedical Engineering at the University of California, Irvine. From 2001 to 2008, he worked at the Beckman Laser Institute and Medical Clinic at UC Irvine. He joined the faculty of UNIST in 2012 as an assistant professor for School of Nano-Bioscience and Chemical Engineering. His research focus is to develop new optical technologies that address challenges in clinical medicine and basic biological research. The long-term goal is to pursue implementation of research tools for in vivo translational studies, where functionalities such as real-time measurement, compactness, and portability are essential.

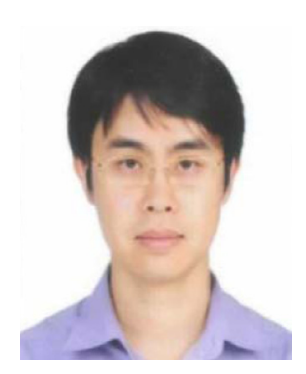

Jeehyun Kim received his $\mathrm{PhD}$ degree in biomedical engineering from University of Texas at Austin in 2004. He is a professor in the School of Electrical Engineering, College of IT Engineering of Kyungpook National University, Daegu, South Korea. He is a member of The Optical Society of Korea and IEEE. His current research interests include optical coherent tomography, Brillouin optical time domain reflectometry, and multiphoton microscopy.

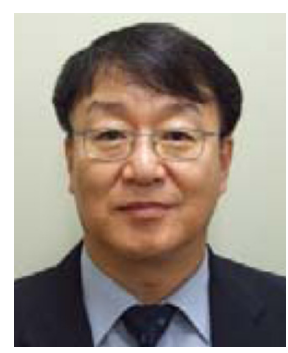

Shinwon Kang received his $\mathrm{PhD}$ degree in biomedical science from the University of Keio in 1993. He is a professor in the School of Electrical Engineering, College of IT Engineering of Kyungpook National University, Daegu, South Korea. He has founded DTEC (Display Technology Educational Center) in the campus, as a chief of the center, from 2003 up to now. His current research interests include opto-electronic functional devices, organic light emitting diodes, and nanorod devices. 